Rev Inv Vet Perú 2002; 13 (2): 44-50

\title{
INFECCIÓN POR Leishmania sp. EN CANINOS DEL DISTRITO DE PAMPAS GRANDE, ANCASH
}

\author{
Guicela Medina R. ${ }^{1}$, Amanda Chávez V. ${ }^{2}$, Gloria Minaya G. ${ }^{3}$, Elizabeth G.O. \\ Barbosa-Santos ${ }^{4}$ y Gloria Espinoza $\mathbf{Z}^{5}$
}

\section{A BSTRACT}

The Andean Leishmaniasis locally known as "uta" is the most frequent type of cutaneous leishmaniasis in Peru. Ancash is an endemic region and Pampas Grande is the district with higher incidence of the disease in humans in the province of Huaraz in 1998. The objective of this research was to determine the presence of Leishmania sp. infection in domestic dogs in the district of Pampas Grande. A total of 83 dogs distributed in seven villages were sampled in August-September 2000. All samples were submitted to three laboratory analyses for the detection of cutaneous leishmaniasis: Giemsa smears, antibody detection in serum through indirect immunofluoresence (IFI), and immunoleish skin test (IDR). The results indicate 3.6\% incidence while using Giemsa smears and IFI, whereas $4.8 \%$ while using IDR. The study showed that Leishmania sp. infection is present in at least $5.4 \%$ of the canine domestic dog population in Pampas Grande.

Key words: uta, canine leishmaniasis, indirect immunofluorescence test, canine's skin test, smears, Pampas Grande

\section{Rrauman}

La leishmaniasis andina, comúnmente conocida como "uta" es la forma más frecuente de presentación de leishmaniasis cutánea en el Perú. El departamento de Ancash es una zona endémica de la enfermedad y Pampas Grande el distrito con mayor incidencia de casos humanos observados en la provincia de Huaraz en el año 1998. El objetivo del presente trabajo fue determinar la presencia de infección por Leishmania sp. en los caninos domésticos del distrito de Pampas Grande. Un total de 83 perros provenientes de 7 caseríos, fueron muestreados entre agosto y septiembre de 2000 . Estos animales fueron sometidos a 3 pruebas diagnósticas de leishmaniasis cutánea: frotis-coloración Giemsa, determinación de anticuerpos en suero mediante inmunofluorescencia indirecta (IFI) e intradermorreacción canina (IDR). Los resultados indicaron 3.6\% de animales positivos a frotis-coloración Giemsa e IFI, y 8.4\% de animales reactores positivos a IDR. Se concluye que la infección por Leishmania sp. en perros del distrito de Pampas Grande está presente en al menos $5.4 \%$ de la población canina del lugar.

Palabras clave: uta, leishmaniasis canina, inmunofluorescencia indirecta, intradermoreacción canina, frotis, Pampas Grande

\footnotetext{
${ }^{1}$ Práctica privada. E-mail: guicelamedina@yahoo.com

${ }^{2}$ Laboratorio de Microbiología y Parasitología, FMV-UNMSM. E-mail: a_chavez_g@hotmail.com

${ }^{3}$ INS, Laboratorio de Leishmania y Chagas

${ }^{4}$ Fundaçao Oswaldo Cruz, Escola Nacional de Saude Publica

${ }^{5}$ DIRESA-Huaraz. Hospital Regional de Huaraz
} 


\section{INTRODUCCIÓN}

La leishmaniasis es endémica en los valles interandinos, así como en el llano amazónico del Perú. Se presentan dos formas principales de leishmaniasis tegumentaria, definidas principalmente por características geográficas y clínicas; la leishmaniasis andina o uta, y la leishmaniasis selvática o espundia (Arias et al., 1996).

La uta se presenta en las laderas occidentales de los valles andinos e interandinos, entre los 800 y $3,000 \mathrm{msnm}$ donde la población se dedica principalmente a la agricultura y ganadería en pequeña escala. La ausencia de verdaderos bosques en las localidades peruanas donde la leishmaniasis tegumentaria es endémica, probablemente constituiría una de las características epidemiológicas que más la diferenciaría de la leishmaniasis tegumentaria existente en los demás países sudamericanos (Arias et al., 1996; OPS, 1994; Herrer, 1951a; Herrer, 1951c).

Esta enfermedad va en aumento en el país. La incidencia anual por detección pasiva en 1978 fue de 9.1 casos por cada 100,000 habitantes, y para 1992 fue de 15,000 nuevos casos. Investigaciones realizadas en áreas de leishmaniasis cutánea proponen como reservorios de la uta a pequeños roedores silvestres y a caninos domésticos. Estudios realizados en el Perú sostienen que el perro podría actuar como reservorio sinantrópico secundario de la uta (OMS, 1984; Llanos-Cuentas et al., 1999; Soulsby, 1987). En este sentido, Llanos-Cuentas et al. (1999) aislaron Leishmania peruviana de aspirados nasales y de biopsias de perros asintomáticos en villas endémicas de los andes peruanos y encontraron sangre de perro en los intestinos de los mosquitos vectores Lutzomyia peruensis y Lutzomyia verrucarum capturados dentro de las casas.

Pirmez et al. (1988) infectaron perros con promastigotes de Leishmania braziliensis (provenientes de lesiones en pe- rros naturalmente infectados) encontrando que las lesiones desarrolladas en la leishmaniasis tegumentaria canina experimental son similares a las lesiones de leishmaniasis cutánea humana. Estas lesiones comienzan con la formación de una pápula, la cual incrementa su tamaño y finalmente se ulcera.

Debido a la presentación de un elevado número de casos nuevos de leishmaniasis cutánea en humanos reportados en el distrito de Pampas Grande en 1998; así como a las evidencias sobre la presencia del parásito en los caninos domésticos en otras localidades con características epidemiológicas muy similares, se planteó realizar el presente estudio a fin de determinar la presencia de la infección por Leishmania sp. en los caninos domésticos de la zona.

\section{Material y Métodos}

\section{Lugar de Estudio}

El presente trabajo se realizó en el distrito de Pampas Grande, provincia de Huaraz, localizado a $450 \mathrm{~km}$ de la ciudad de Huaraz. Posee una extensión de $8,000 \mathrm{~km}^{2}$, y una altitud que oscila entre los 1,000 y los 3,800 msnm. El distrito está dividido en 7 caseríos: Pampas, Matara, Querway, Cullash, Poctas, Shankak y La Victoria. Cuenta con una población de 1,505 habitantes, cuyas edades fluctúan entre los 0-70 años; de éstos, 628 (42\%) son menores de 18 años y $357(24 \%)$ son mujeres entre los 15-49 años (DIRESAChavín, 2000).

La topografía del distrito de Pampas Grande es bastante accidentada, cuenta con valles y montañas íntegramente dedicadas a la pequeña ganadería (vacunos, ovinos, mulares) y a la pequeña agricultura de artículos de pan llevar (habas, alfalfa, trigo, papa, etc.), con mucha dependencia del clima. Sus caminos son rurales y el desplazamiento de los pobladores es a pie, a caballo o en burro; 
con un promedio de 4 a 6 horas entre uno y otro caserío. Esta descripción topográfica coincide con la que caracteriza a zonas utógenas (Herrer, 1951c).

\section{Tamaño Muestreal}

El tamaño de la muestra se calculó teniendo en cuenta la prevalencia referencial de $5.4 \%$ obtenida en estudios anteriores en áreas endémicas de los Andes peruanos (Llanos-Cuentas et al., 1999), y se utilizó la fórmula de prevalencia límite (Thrusfield, 1990).

Se muestrearon 83 canes domésticos mestizos de ambos sexos, a lo largo de los 7 caceríos del distrito de Pampas Grande. Los animales tenían edades comprendidas entre los 6 meses y 12 años, y poseían un historial de permanencia en la zona no menor de 3 meses. Se confeccionó una ficha epidemiológica a cada animal.

\section{Recolección de Muestras y Proce- samiento}

El muestreo se realizó durante los meses de agosto a septiembre de 2000 . A cada animal se le hizo un examen clínico externo buscando lesiones cutáneas activas o cicatriciales sugestivas de leishmaniasis cutánea; tales como úlceras, despigmentaciones o cicatrices antiguas con diámetro $\geq 0.5 \mathrm{~cm}$ en cualquier parte del cuerpo y nódulos en áreas con poco pelo (Falqueto $e t$ al., 1986; Bonfante-Garrido et al., 1981). Se obtuvo una muestra del lugar de la lesión, se realizó un frotis de nariz y boca, y se colectó una muestra de sangre para la investigación serológica por inmunofluorescencia indirecta (IFI). Además se realizó la prueba de intradermorreacción (IDR). Los frotises fueron coloreados con Giemsa. La observación de amastigotes fue considerada como resultado positivo.

Para la prueba de IDR, se siguieron las indicaciones de Marzochi y Barbosa Santos (1998) y Bonfante-Garrido et al. (1981), inyectando por vía intradérmica $0.1 \mathrm{ml}$ de inmunoleish P $10000 \mathrm{G}$ (donado por la ENSP Oswaldo Cruz y elaborado con promastigotes de Leishmania braziliensis). Se consideró IDR positiva si uno de los diámetros de la induración era $\geq 5 \mathrm{~mm}$ (OMS, 1984). En el caso de IFI, se consideró como positivos a los sueros que dieron una reacción fluorescente a partir de una dilución de 1/40 (Barbosa-Santos et al., 1998).

\section{Resultados}

El cuadro 1 muestra los resultados de la frecuencia de animales positivos a las pruebas de frotis, IDR e IFI, encontrándose 3.6\% $(3 / 83), 8.4 \%$ (7/83) y $3.6 \%$ (3/83) de animales positivos, respectivamente. Haciendo una comparación entre los resultados obtenidos con las pruebas de frotis e IDR mediante las pruebas estadísticas de Kappa y Mc Nemar, se obtuvo una concordancia de $58 \%$, indicando que las pruebas no son mutuamente reemplazables. Resultados similares se obtuvieron al comparar los resultados de las pruebas de IFI e IDR. Sin embargo, al comparar los resultados obtenidos con las pruebas de frotis e IFI, se encontró que existe una concordancia de $100 \%$, siendo las pruebas mutuamente reemplazables.

No se detectaron lesiones cutáneas activas de leishmaniasis cutánea (uta) en ninguno de los animales muestreados al examen clínico; sin embargo, 2 de los 3 animales positivos a las 3 pruebas diagnósticas mostraron lesiones cicatriciales en la nariz, sugestivas de leishmaniasis cutánea (Cuadro 2).

\section{Discusión}

La leishmaniasis cutánea, conocida como uta, es una enfermedad de frecuente presentación en los residentes del distrito de Pampas Grande (Huaraz). La presencia de la enfermedad en este lugar en particular, 
Cuadro 1. Frecuencia de canes positivos a Leishmania sp. para las pruebas diagnósticas de Frotis, Intradermorreacción canina, (IFI) e Inmunofluorescencia Indirecta (IDR) en el distrito de Pampas Grande, Huaraz. Agosto-setiembre de 2000

\begin{tabular}{lccccccc}
\hline \multirow{2}{*}{ Cacerío } & \multirow{2}{*}{$\begin{array}{c}\mathrm{N}^{\mathbf{0}} \\
\text { perros }\end{array}$} & \multicolumn{2}{c}{ Frotis } & \multicolumn{2}{c}{ IDR } & \multicolumn{2}{c}{ IFI } \\
\cline { 3 - 9 } & & $\mathrm{n}$ & $\%$ & $\mathrm{n}$ & $\%$ & $\mathrm{n}$ & $\%$ \\
\hline Pampas & 16 & 1 & 6.3 & 2 & 12.5 & 1 & 6.3 \\
Matara & 13 & 0 & 0.0 & 2 & 15.4 & 0 & 0.0 \\
Querway & 08 & 0 & 0.0 & 0 & 0.0 & 0 & 0.0 \\
Poctas & 10 & 0 & 0.0 & 0 & 0.0 & 0 & 0.0 \\
Cullash & 11 & 1 & 9.1 & 1 & 9.1 & 1 & 9.1 \\
Shankak & 10 & 0 & 0.0 & 1 & 10.0 & 0 & 0.0 \\
La Victoria & 15 & 1 & 6.7 & 1 & 6.7 & 1 & 6.7 \\
\hline Total & 83 & 3 & 3.6 & 7 & 8.4 & 3 & 3.6 \\
\hline
\end{tabular}

Cuadro 2. Distribución de los canes positivos a Leishmania sp., según las pruebas diagnósticas de Frotis, Inmunofluorescencia indirecta (IFI) e Intradermoreacción (IDR) en el distrito de Pampas Grande, Huaraz. Agosto-Setiembre del 2000

\begin{tabular}{lccccccc}
\hline Cacerío & Lesión & Frotis & IDR & $\begin{array}{c}\text { Diámetro de la } \\
\text { induración } \\
\text { IDRM (mm) }\end{array}$ & IFI & $\begin{array}{c}\text { Título en } \\
\text { Sueros } \\
\text { IFI }\end{array}$ & $\begin{array}{c}\mathrm{N}^{\circ} \text { de pruebas } \\
\text { positivas }\end{array}$ \\
\hline Pampas & NO & - & + & 5 & - & $<1 / 40$ & 1 \\
Pampas & NO & + & + & 9 & + & $1 / 160$ & 3 \\
Matara & NO & - & + & 8.5 & - & $<1 / 40$ & 1 \\
Matara & NO & - & + & 5 & - & $<1 / 40$ & 1 \\
Cullash & Sí* & + & + & 12 & + & $1 / 320$ & 3 \\
Shankak & NO & - & + & 7 & - & $<1 / 40$ & 1 \\
La Victoria & Sí* & + & + & 10 & + & $1 / 160$ & 3 \\
\hline
\end{tabular}

* Presencia de lesiones cicatriciales en nariz

+ Positivo; - negativo

obedece a que se encuentran presentes todos los elementos estructurales necesarios para el desarrollo de un foco "utógeno"; es decir, presenta condiciones geográficas adecuadas para el desarrollo de las especies de flebótomos agentes de transmisión, y se tienen los hospederos reservorios mamíferos, así como las formas de la enfermedad en los microorganismos causantes.

Según Minaya (1997), para diagnosticar la enfermedad se requiere el resultado positivo de dos pruebas diferentes como mínimo. Así, el hallazgo de Leishmania sp. en el $3.6 \%$ de los perros evaluados a través de las tres pruebas diagnósticas utilizadas, confirma la presencia de la infección parasitaria en la población canina del lugar. Los resultados de las pruebas de IDR y frotis no coincidieron en el total de los casos, detectándose en ocasiones animales positivos a IDR únicamente. La IDR detecta pacientes con enfermedad activa que tienen más de $\mathbf{4}$ semanas de evolución y personas con infección 
antigua o cicatrizada, mientras que el frotis indica la presencia o ausencia de parásitos en las lesiones de pacientes con enfermedad activa principalmente, ya que en infecciones antiguas generalmente sólo está presente la lesión, pero no el parásito (Minaya, 1997).

El hecho de que se encuentren reacciones positivas a la prueba de IDR hace suponer que estos animales estuvieron en algún momento de su vida en contacto con el parásito. Además, el hallazgo de anticuerpos en la IFI y parásitos presentes en el frotis de un perro que no mostró ninguna lesión aparente activa o cicatricial ni ha mostrado anteriormente signos de la enfermedad según indica su propietario, demuestra que en la zona del presente estudio, la uta en los canes sería en algunos casos de tipo subclínico y estos animales podrían actuar como hospederos sinantrópicos secundarios.

Estudios previos realizados por LlanosCuentas et al. (1999) y Herrer (1951b) demuestran la presencia del parásito en pequeños roedores como Didelphis albiventris, Phyllotis andinum y Akodon sp., y en perros y zorros en zonas utógenas del Perú con características epidemiológicas similares a las observadas en Pampas Grande. Asimismo, investigaciones en otras partes del continente como Brasil (Sales-Barbosa et al., 1999; Barbosa-Santos et al., 1998; Maywald et al., 1993; Falqueto et al., 1986; Pirmez et al., 1988; Coutinho et al., 1985) y Venezuela (Bonfante-Garrido et al., 1981) demuestran la presencia y el papel que juegan los canes en la epidemiología de la leishmaniasis cutánea. Sin embargo, cabe aclarar que en esos países existen áreas donde además de presentarse leishmaniasis cutánea, también se encuentra la leishmaniasis visceral.

El $3.6 \%$ de positividad en perros del distrito de Pampas Grande concuerda con los resultados obtenidos por Llanos-Cuentas $e t$ $a l$. (1999) en otras zonas del país donde existen casos de uta en humanos (Ancash, provincia de Bolognesi, $4.5 \%$; y Lima, provincia de Canta, $4.5 \%$ ); pero difiere de estudios efectuados en perros de otros países como Brasil (25.5\%; Barbosa-Santos et al., 1998) y Venezuela (36.4\%, Bonfante-Garrido et al., 1981), lo que puede deberse a la forma de presentación de la enfermedad canina en el Perú, donde los perros usualmente tienen lesiones cutáneas poco notorias o de tipo subclínico (Herrer, 1951b). Esto es contrario a lo que ocurre con la leishmaniasis cutánea en Brasil, Argentina y Venezuela donde la enfermedad en perros es más manifiesta, lo que puede atribuirse a las características propias de cada especie de leishmania. Sin embargo, la ausencia de lesiones sospechosas en perros provenientes de áreas endémicas de leishmaniasis cutánea humana no significa que la enfermedad canina esté ausente (Cardoso et al., 1989).

Los probables vectores en la zona de estudio son Lutzomyia peruensis y Lutzomyia verrucarum que tienen hábitos intra y peridomiciliarios, y su alimentación es de tipo antropofílico y zoofílico, por lo que no es raro hallarlos en las viviendas del lugar. En estudios recientes se encontró que en algunas zonas del departamento de Ancash, el Lutzomyia peruensis representa el $51 \%$ de los flebótomos capturados en el interior de las viviendas y el $85 \%$ del total capturado con cebos humanos (Arias et al., 1996; Cáceres, 1996). Todas estas variables probablemente pueden tener una fuerte influencia sobre el rol de los perros en la epidemiología de la enfermedad de acuerdo con cada una de las áreas específicas. Sin embargo, queda mucho por esclarecer aún con relación al rol de los canes y otros mamíferos domésticos y silvestres como posibles hospederos reservorios.

\section{Conclusiones}

El presente trabajo permite concluir que en el periodo 1999-2000 la leishmaniasis cutánea causada por Leishmania sp. estuvo presente en el $5.4 \%$ de la población canina del distrito de Pampas Grande, Huaraz. 


\section{Limeratura Citada}

1. Arias, J.; F. Beltrán; P. Besjeux. 1996. Epidemiología y control de la leishmaniasis en las Américas, por país o territorio. Cuaderno Técnico $N^{\circ} 44$. OPS. Washington.

2. Barbosa-Santos, E.G.O.; M.C.A. Marzochi; N.F. Conceicao; C.M.M. Brito; R.S. Pacheco. 1998. Epidemiological survey on canine population with the use of immunoleish skin test in endemic areas of human american cutaneous leishmaniasis in the State of Rio de Janeiro, Brazil. Rev. Inst. Med. Trop. S. Paulo 40: 41-47.

3. Bonfante-Garrido, R.; N. Morillo; $R$. Torres. 1981. Leishmaniasis cutánea canina en Venezuela. Bol. Of. Sanit. Panam. 91: 160-165.

4. Cáceres, A. 1996. Especies de lutzomyias vectores de la uta en el Perú. Revista Peruana de Entomología 38: 23-26.

5. Cardoso, S.R.A.; M.E. Machado; J.M.C. Cruz; M.R.E. Goncalves; W.H. Stutz. 1989. Leishmaniose tegumentar canina no município de Uberlandia, Minas Gerais: Diagnóstico clínico e sorológico de caes naturalmente infectados. Rev. Centenaria. Centifica. Biomedica. Universidad Federal de Uberlandia 5: 14-21.

6. Coutinho, S.; M.P. Nunes; M.C.A. Marzochi; N. Tramontano. 1985. A survey for american cutaneous and visceral leishmaniasis among 1342 dogs from areas in Rio de Janeiro (Brazil) where the human diseases occur. Mem. Inst. Oswaldo Cruz, Rio de Janeiro 80: 17-22.

7. DIRESA-Chavín, Estadística e Informática. 2000. Estimación de la población por grupos de edad según provincias, distritos y establecimientos de salud. DIRES-Ancash.

8. Falqueto, A.; J. Rodrigues; G. Coutinho; G. Grimaldi; P.A. Sessa; V.R. Daher; A. Claudino de Jesús; J. Tasso. 1986. Participacao do cao no ciclo de transmissao da leishmaniose tegumentar no Municipio de Viana, Estado do Espirito Santo, Brasil. Mem. Inst. Oswaldo Cruz. Rio de Janeiro 81: 155163.

9. Herrer, A. 1951a. Estudios sobre leishmaniasis tegumentaria en el Perú. IV. Observaciones epidemiológicas sobre la uta. Rev. Med. Exp. (Lima) 8: 45-83.

10. Herrer, A. 1951b. Estudios sobre leishmaniasis tegumentaria en el Perú. V. Leishmaniasis natural en perros procedentes de localidades utógenas. Rev. Med. Exp. (Lima) 8: 87-118.

11. Herrer, A. 1951c. Estudios sobre la leishmaniasis tegumentaria en el Perú. VI. Relación entre leishmaniasis tegumentaria y phlebotomus. Rev. Med. Exp. (Lima) 8: 119-134.

12. Llanos-Cuentas, E.; N. Roncal; P. Villaseca; L. Paz,; E. Ogusuku; J.E. Perez; A. Caceres; C. Davies. 1999. Natural infections of Leishmania peruviana in animals in the Peruvian Andes. Trans. Roy. Soc. Trop. Med. Hyg. 93: 15-20.

13. Marzochi, M.C.A.; E.G.O. BarbosaSantos, 1988. Evaluation of a skin test on the canine mucocutaneous leishmaniasis diagnosis. Mem. Inst. Oswaldo Cruz 83: 391-392.

14. Maywald, P.G.; M.I. Machado; J.M. Costa; M.G. Oliveira; M.R.F.G. Pires. 1993. Canine cutaneous leishmaniosis: seroepidemiological inquiry in urban and rural area of Uberlandia, Minas Gerais, Brazil. Braz. J. Vet. Res. Anim. Sci. Sao Paulo 30: 25-29.

15. Minaya, G. 1997. Manual de procedimientos para el diagnóstico de leishmaniasis. Serie de Normas Técnicas $\mathrm{N}^{\circ} 13$. $2^{a}$ ed. p 11-39. MINSA. Perú.

16. OMS. Organización Mundial de la Salud. 1984. Las leishmaniasis. Serie de Informes Técnicos $\mathrm{N}^{\circ}$ 701. p 70-151. Ginebra, Suiza.

17. OPS. Organización Panamericana de la Salud. 1994. Las leishmaniasis en las Américas. Boletín Epidemiológico. OPS 15: 12-13. 
18. Pirmez, C.; M.C.A. Marzochi; S.G. Coutinho. 1988. Experimental canine mucocutaneous leishmaniasis (Leishmania braziliensis braziliensis). Mem. Inst. Oswaldo Cruz. Rio de Janeiro 83: 145-151.

19. Sales-Barbosa, G.M.; M.C. Marzochi; C.L. Massard; G. Pires; E. Mouta. 1999. Aspectos epidemiologicos da leishmaniose tegumentar americana em caes, no Municipio de Paraty, Estado do Rio de Janeiro, Brasil. Cad. Saude Publica, Rio de Janeiro 15: 641-646.

20. Soulsby, E.J.L. 1987. Parasitología y enfermedades parasitarias en los animales domésticos. $7^{\mathrm{ma}}$ ed. Nueva Editorial Interamericana. Mexico D.F. 823 p.

21. Thrusfield, M. 1990. Epidemiología Veterinaria. Ed. Acribia. Zarágoza, España. 